\title{
Spontaneously Ruptured Endometriotic Cysts-A Case Report
}

\author{
Dr Shradha Pal*, Dr. Mrinalini Sahasrabhojanee** \\ *Dr. Shradha Pal, Senior resident, Department of Obstetrics and Gynaecology Goa Medical College \\ **Dr.Mrinalini Sahasrabhojanee, Assistant Professor, Department of OBG Goa Medical College \& hospital
}

\section{Introduction}

Endometriosis is one of the most common conditions encountered in gynaecological practice today and yet continues to be an enigma. Endometriosis occurs in about $10 \%$ of women of reproductive age and carries an infertility rate of up to $50 \%$. Besides being associated with infertility and chronic pelvic pain it is now an emerging cause of gynecological emergencies.

\section{Case Report}

A 24 year old nulligravida presented to the Goa Medical College with chief complaints of noticing mass per abdomen since two months and progressively worsening dysmenorrhoea since one year on 8/7/2015. She was afebrile, normotensive with no tachycardia and per abdomen she had a mass palpable reaching up to the umbilicus which was cystic in consistency and non tender.

She presented with severe abdominal pain and vomiting in a peripheral health centre on 14/7/2015. Subsequent to this episode, the patient continued to have dull aching pain which was however significantly less. She then presented to our OPD a week later wherein there was a significant reduction in the size of the ovarian mass and surprisingly no signs of acute abdomen!

The preoperative investigations done showed haemoglobin of $11.2 \mathrm{~g} \%$ (before and after the rupture).the patient had a total count of 7,200 and a CA 125 of $3000.2 \mathrm{IU} / \mathrm{ml}$ preoperatively. The ultrasonography of the abdomen and pelvis done after suspected rupture on 22/7/15 showed a large cyst $11 * 7.3 * 7.2 \mathrm{~cm}$ with thick walls and densely packed internal echoes noted in the right ovary. The uterus and left ovary were normal. There was no evidence of hemoperitoneum. There was a reduction in the size of the cyst however compared to the prior USG.

The patient underwent a laparoscopic bilateral endometriotic cyst excision with ablation of the endometriotic deposits with adhesiolysis under general anaesthesia on 24/7/2015.

The intraoperative findings were suggestive of a ruptured right sided endometriotic cyst $8 * 10 \mathrm{~cm}$, adherent to the posterior surface of the uterus and the pouch of Douglas. Spillage of chocolate colored fluid was noted over the entire peritoneal cavity including the undersurface of the diaphragm as well as the omentum. Surprisingly there was no hemoperitoneum. A $4 * 3 \mathrm{~cm}$ left sided endometriotic cyst was also present. The uterus was retroverted.

Subsequently, the histopathological report revealed an endometriotic cyst.

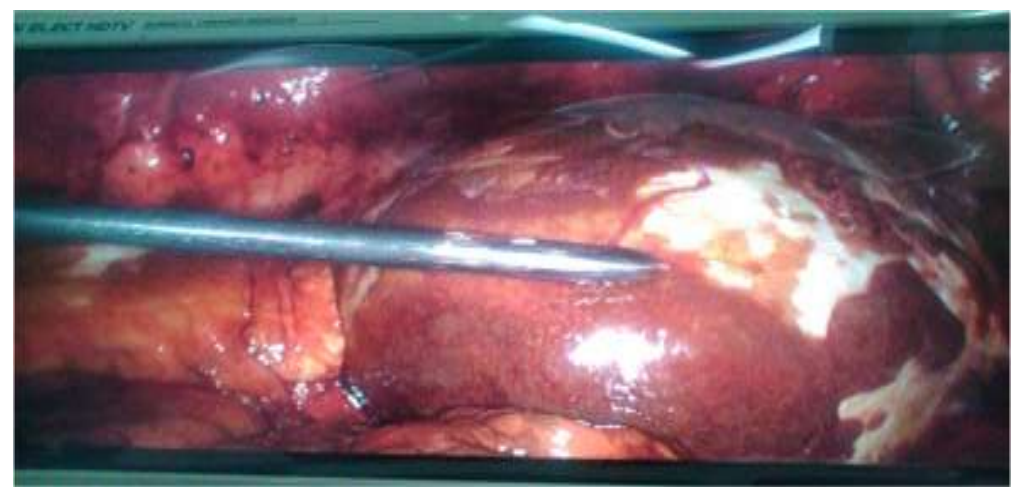

A picture showing the ruptured right sided endometriotic cyst 

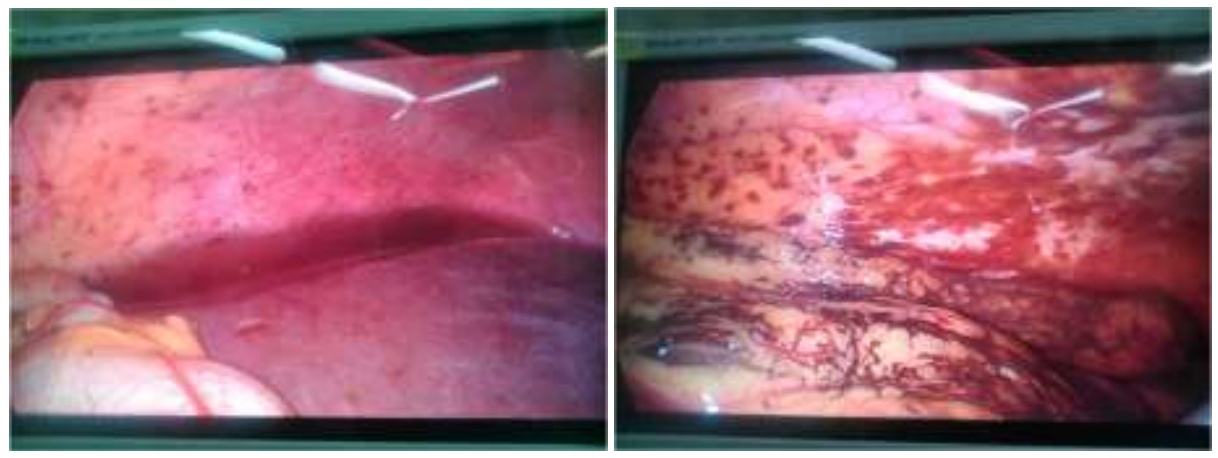

Endometriotic deposits over the entire peritoneal cavity, undersurface of diaphragm and viscera A week later we had another different presentation of a ruptured endometriotic cyst. A 28 year old nulligravida married since 5 years with primary infertility presented on 29/7/2015 with acute abdomen. On examination she had tachycardia though normotensive and per abdomen had guarding and rigidity. Radiological findings revealed a left sided ovarian cyst $6 * 5 \mathrm{cms}$ with dense internal echoes and hemoperitoneum. USG guided preoperative tapping done revealed chocolate colored fluid.

An emergency laparotomy with left sided ovarian cystectomy with ovarian reconstruction and peritoneal lavage was done for the same.

Intraoperative findings showed a left sided ruptured endometriotic cyst $5 * 5 \mathrm{cms}$ with extensive adhesions of the same with the posterior surface of the uterus. $300 \mathrm{cc}$ of chocolate colored fluid was aspirated from the peritoneal cavity admixed with blood. Histopathological examination revealed endometriotic cyst.

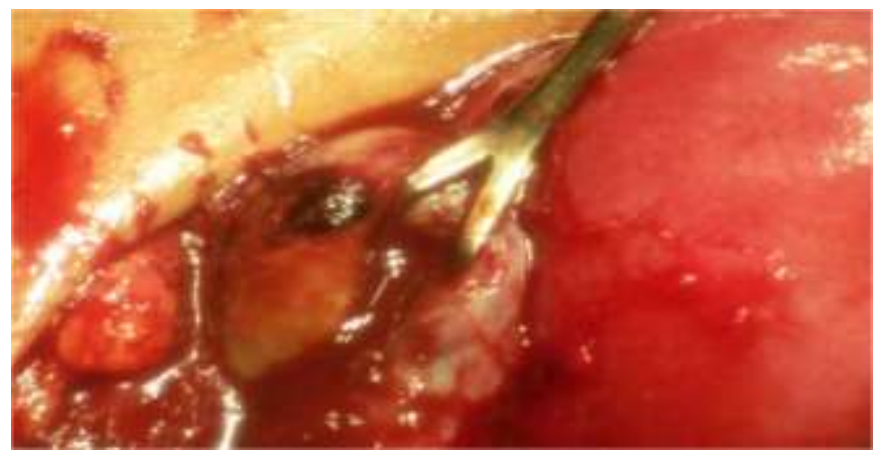

\section{Discussion}

Women with a history of endometriosis may carry an elevated risk for hemorrhagic complications, but enlargement and rupture seldom occur ${ }^{2}$.It is acceptable that large endometriomas of $6 \mathrm{~cm}$ or more in diameter may carry additional risks, including increased likelihood of rupture, infection or presence of malignancy nevertheless, they may be polycystic and represent an endometrioma with a communicating or noncommunicating luteal cyst, as observed by Sampson (1921) in 9\% of his cases ${ }^{3}$. During the last decade, the increased use of assisted reproductive technologies has led to higher fertility rates in patients with endometriosis and to a higher incidence of multiple gestations.

Therefore, the number of pregnant women with endometriosis and associated complications may rise. Ueda et al. in 2010 reported an incidence of $0.52 \%$ for ovarian endometriotic mass during pregnancy. ${ }^{4,5}$

Evangelinakis et al.2009 calculated that the incidence of hemoperitoneum caused by ruptured endometriotic cysts in a group of nonpregnant women in reproductive age is $2.22 \%$. Serum CA-125 measurement is now a consolidated method for diagnosing endometriosis, but its interpretation has posed a number of problems, particularly its utility in diagnosing minimal/mild endometriosis, whereas its value as a diagnostic aid in the moderate-severe stages is well recognized. The serological testing for CA-125 has been widely used not only to detect endometriosis, but also to monitor its progression ${ }^{4,5}$.

However, endometriosis constitutes a major non-malignant gynaecological disease wherein the serum CA-125 levels are in the malignancy range $(>1,000 \mathrm{IU} / \mathrm{ml})^{6}$. The CA-125 levels are reported to rise immediately after the rupture of an endometriotic cyst and also following malignant transformation ${ }^{7}$. As in our case the CA-125 levels were as high as $3000.2 \mathrm{IU} / \mathrm{ml}$ after the rupture. An explosive rise of the serum CA-125 levels to up to $9300 \mathrm{IU} / \mathrm{ml}$ following the rupture of ovarian endometrioma has been reported ${ }^{8}$.

The high CA-125 levels in our case did raise a suspicion of malignancy however the radiologic features were that of a benign cyst and so was the histopathological report. 
Similar cases have been reported in literature. A $35 \mathrm{yr}$ old lady, para 2, living 2, with 2 previous caesarean sections, presented with the features of acute abdomen, markedly raised CA125 levels (7910 $\mathrm{IU} / \mathrm{ml}$ )and bilateral ruptured ovarian endometriomas. ${ }^{9}$ Several cases of ruptured endometriotic cysts with hemoperitoneum in pregnancy have been reported especially in twin gestations due to the enlarging uteri displacing the ovarian cysts ${ }^{10}$.

\section{Conclusion}

Ovarian endometrioma and endometriosis may present acutely in varied ways and they may be associated with extremely elevated serum CA-125 levels. For this reason, ovarian endometrioma should be considered with respect to the differential diagnosis of reproductive-age women who present with an acute abdomen and an ovarian mass, even if it resembles an ovarian malignancy. Moreover, very high CA-125levels do not necessarily forebode an ovarian malignancy.

\section{References}

[1]. Holoch KJ, Lessey BA. Endometriosis and infertility. Clin Obstet Gynecol 2010;53:429-38.

[2]. Philipp Reif et al, Rupture of endometriotic cyst causes acute abdomen in twin pregnancy. Fertility and Sterility Vol. 95, No. 6, May 2011

[3]. I Brosens et al, Management of the ovarian endometrioma. Reproductive BioMedicine Online (2014) 28, 232- 238. Cheng YM, Wang ST, Chou CY. Serum CA-125 in preoperative patients who are at a high risk for endometriosis. Obstet Gynecol. 2002; 99 (3):375-80.

[4]. Rosa E Silva AC, Rosa E Silva JC, Ferriani RA. Serum CA-125 in the diagnosis of endometriosis. Int J Gynaecol Obstet. 2007; 96:206-7.

[5]. He RH, Yao WM, Wu LY, Mao YY. Highly elevated serum CA-125 levels in patients with non-malignant gynecological diseases. Arch Gynecol Obstet. 2011;283:107-10.

[6]. Check JH. CA-125 as a biomarker for the malignant transformation of endometriosis. Fertil Steril. 2009; $91:$ e35; author reply e36. Epub 2009 Mar 31.

[7]. Johansson J, Santala M, Kauppila A. The explosive rise of serum CA 125 following the rupture of ovarian endometrioma. Hum. Reprod 1998; 13:3503-3504.

[8]. Kahraman K, Ozguven I, Gungor M, Atabekoglu CS. Extremely elevated serum CA-125 levels as a result of unruptured unilateral endometrioma: the highest value reported. Fertil Steril. 2007; 88: 968.

[9]. Ruptured bilateral endometriotic cysts with markedly elevated CA-125 levels. Anagha K. Journal of Clinical and Diagnostic Research. 2011 October, Vol-5(5): 1109-1110

[10]. Phillip Reif et al.rupture of endometriotic cyst in a late twin gestation.Vol 95.n0.6,Fertility and Sterility,May 2011. 